\title{
Scrivere la storia dell'arte: metodologia e ricerca negli ultimi decenni
}

\author{
Carla Subrizi \\ Università degli Studi di Roma La Sapienza \\ carla.subrizi@uniroma1.it
}

RESUMEN: All'inizio del nuovo secolo la questione della storia del'arte e dei metodi da utilizzare per la ricerca riapre molti interrogativi e determina la necessità di una revisione critica della storiografia del passato XX secolo. Molte iniziative dimostrano la necessità che molti storici dell'arte, nelle Università italiane ma anche internazionali avvertono in questi anni: i dipartimenti di storia dell'arte, nelle differenti denominazioni che hanno in Europa e in altri paesi del mondo, concentrano ricerche e iniziative sullo stato della storia dell'arte, su quanto sia necessario rivedere e interrogare le strategie della sua costruzione, nonché l'identificazione dei documenti e materiali d'archivio considerati le sue «fonti». Tali problemi coinvolgono gruppi di lavoro e ricerche differentemente orientati, all'interno dei quali è tuttavia possibile individuare premesse simili che da una parte affrontano la questione su un piano teorico e metodologico, dall'altro si concentrano su casi di studio, su particolari aspetti storici e filologici, per mettere in pratica, nuove ipotesi di studio e ricerca. II saggio si sofferma su tali questioni e analizza alcuni passaggi fondamentali della bibliografia più recente.

PALABRAS CLAVE: Arte contemporanea; Storia dell'arte; Canone; Storiografia; Narrazione.

\section{Writing the History of Art: Methodology and Research in the Last Decades}

ABSTRACT: At the beginning of the new century the issue of art history and the methods to be used for research reopens many questions and determines the need for a critical review of historiography of the $20^{\text {th }}$ century. Numerous initiatives demonstrate this need that many art historians in both Italian and international universities have felt in recent years. Art history departments, in their different denominations in Europe and other countries around the world, are concentrated on research and initiatives on the state of art history, on how to revise and question the strategies of its construction, and on the identification of documents and archival material considered its «sources". These problems involve work groups and differently oriented researches within which, however, it is possible to identify similar premises. On the one hand they address the issue on a theoretical and methodological level and on the other, focus on case studies regarding particular historical and philological aspects in order to put new hypotheses of study and research into practice. The essay focuses on these issues and looks at some of the basic segments in the most recent bibliography.

KEYWORDS: Contemporary Art; Art History Canon; Historiography; Narrative.

Recibido: 25 de abril de 2017 / Aceptado: 4 de septiembre de 2017.

Un progetto di ricerca, dal titolo La scrittura delle storie, da me stessa avviato nel 2007 con un gruppo di docenti della Sapienza Università di Roma, aveva dato inizio a un dialogo a partire dal tema che ci eravamo date e che volevamo affrontare: la discussione di metodi e questioni inerenti la scrittura della storia (dell'arte, della letteratura, della filosofia) all'inizio del XXI secolo. Avevano aderito al progetto, Silvia Bordini, Luciana Cassanelli, Maria Serena Sapegno, accanto alla partecipazione di studiose internazionali tra le quali Griselda Pollock, Università di Leeds e Maite Méndez Baiges, Università di Malaga. Nel 2010 il progetto fu riconfermato con il titolo Fare storia all'inizio del XXI secolo. La prospettiva adottata era interdisciplinare,

Cómo citar este artículo: SUBRIZI, Carla, "Scrivere la storia dell'arte: metodologia e ricerca negli ultimi decenni», Boletín de Arte-UMA, n. ${ }^{\circ} 38$, Departamento de Historia del Arte, Universidad de Málaga, 2017, pp. 171-178, ISSN: 0211-8483, DOI: http://dx.doi.org/10.24310/BoLArte.2017.v0i38.3362 
tra arte, letteratura, storia sociale dell'arte, tecnica e tecniche artistiche, studi di genere e semiotica, stabilendo relazioni e interazioni tra le discipline e al di fuori di esse.

All'inizio del nuovo secolo la questione della storia e dei metodi da utilizzare riapre molti interrogativi e determina la necessità di una revisione critica della storiografia del passato XX secolo.

Nel 2013, in Italia, la Consulta Universitaria Nazionale per la Storia dell'Arte (Cunsta) organizzava due Giornate di Studio (Milano, Università Cattolica, 28 febbraio-1 marzo) dal titolo Fare la storia dell'arte oggi, durante le quali era affrontato il problema della periodizzazione della storia dell'arte in Italia ovvero come sono nate e come possono essere considerate le date che indicano svolte, inizio e fini di percorsi della storia, momenti cruciali dai quali non si può prescindere. Con il contributo dal titolo La periodizzazione dell'arte italiana del '900, Flavio Fergonzi aveva partecipato al Primo Incontro su La prospettiva storica. Questo delle date e dei periodi che definiscono una svolta o uno sviluppo, un punto di crisi o un cambiamento, costituisce il dato critico, continuamente da rivedere poiché ogni storia dell'arte ha stabilito date e forme di periodizzazione che derivano da modelli, riferimenti, contesti mai tuttavia assoluti e, che invece, appaiono mutevoli, in relazione a mode, tendenze, prospettive internazionali.

Iniziative come queste dimostrano la necessità che molti storici dell'arte, nelle Università italiane ma anche internazionali avvertono in questi anni: i dipartimenti di storia dell'arte, nelle differenti denominazioni che hanno in Europa e in altri paesi del mondo, concentrano ricerche e iniziative sullo stato della storia dell'arte, su quanto sia necessario rivedere e interrogare le strategie della sua costruzione, nonché dell'identificazione dei documenti e materiali d'archivio considerati le sue «fonti». Tali problemi coinvolgono gruppi di lavoro e ricerche differentemente orientati, all'interno dei quali è tuttavia possibile individuare premesse simili che da una parte affrontano la questione su un piano teorico e metodologico, dall'altro si concentrano su casi di studio, su particolari aspetti storici e filologici, per mettere in pratica, nuove ipotesi di studio e ricerca.

La bibliografia che si concentra sulla questione di come la storia e, per quello che ci riguarda, la storia dell'arte si costruisce, è già molto vasta. Faccio riferimento a una bibliografia che negli ultimi anni ha affrontato il problema criticamente: quali storie la storia dell'arte ci racconta, quali nar- razioni vengono costruite, da quale punto di vista e perché, come la storia dell'arte dialoga con altri ambiti disciplinari (antropologia, sociologia, scienze politiche, semiotica, psicoanalisi, ecc.) e come si trasforma e si rinnova attraverso tali relazioni. Inoltre: a quale storia dell'arte ci riferiamo quando diciamo storia dell'arte, in un'epoca, come quella più recente (dalla fine degli anni Ottanta circa) in cui i confini geografici, storici, culturali sono cambiati e in cui si assiste all'emergere di più storie, parallelamente.

Le questioni si estendono poi ad altri aspetti. I metodi e le premesse di quella che è stata la storia dell'arte nel mondo occidentale (tralasciando per ora le differenze anche all'interno di questo panorama) sono confrontati con metodi e premesse propri di paesi non appartenenti al contesto occidentale europeo o nordamericano. Inoltre si cerca di capire come tali metodologie e i risultati stessi di metodologie tradizionali o più innovative ricadano nei progetti di formazione universitari, nelle metodologie della didattica, come anche nell'ambito delle istituzioni o degli allestimenti delle collezioni nei musei.

Nella riconsiderazione dei termini stessi del dibattito, arte e storia, le relazioni tra «canone» e scrittura della storia, tra identità e differenze culturali del pensare e fare la storia, appaiono come i punti fondamentali per un'accurata analisi delle questioni. Altro aspetto riguarda poi, il rapporto che la storia intrattiene con la sua scrittura ovvero se la scrittura debba essere oggettiva, neutra, spersonalizzata dall'autore, dalla sua particolare esperienza. La pratica di porsi come voce attiva nel processo di scrittura sembra non congeniale a premesse che considerano la storia come la costruzione di un panorama oggettivo, al di sopra delle parti e delle voci che concorrono alla sua costruzione.

Il dibattito, e le ipotesi critiche che rispondono o cercano di rispondere a queste questioni, è molto acceso e i testi o le riviste che, solo per darci una data, dagli anni Novanta, hanno presentato aspetti importanti di tali riflessioni, sono moltissimi. Non posso qui soffermarmi su anche sintetici aspetti di questa bibliografia. Vorrei ricordare tuttavia alcune pubblicazioni che hanno costituito, a mio avviso, alcune delle tappe cruciali di questo dibattito, a livello internazionale.

I saggi di Hans Belting, Das Ende der Kunstgeschichte? [La fine della storia dell'arte?] del 1983 (Belting, 1983) e il successivo The Germans and their Art (Belting, 1998), costituiscono una riflessione importante. Belting aveva approfondito i suoi studi di storia dell'arte medioevale prima all'Università 
di Mainz, quindi a Roma presso la Biblioteca Hertziana, poi al centro di ricerca di Dumbarton Oaks a Washington dove aveva incontrato studiosi tra i quali Otto Demus, André Grabar, David Freedberg. Gran parte della sua formazione era però derivata non tanto dalla storia dell'arte quanto dagli studi di filologia, di filosofia e teologia. Ad Amburgo, dove insegna dal 1968, Belting visse in prima persona la disputa avvenuta in seno all'insegnamento della storia dell'arte, in seguito alla quale si delinearono due netti schieramenti: da una parte i sostenitori della traditionelle kunstgeschichte e dall'altra la cosiddetta neue linke, che proponeva invece una riorganizzazione e una ridefinizione della disciplina stessa. Dopo un decennio trascorso all'Università di Heidelberg, nel 1980, Belting si trasferì all'Università Ludwig-Maximilian di Monaco.

In un'intervista, ricordando l'esperienza di Monaco, Belting userà le parole «Katastrophe» e "große Chance», alludendo sì alla fama ma anche ai condizionamenti che implicava la rinomata e radicata tradizione di quella prestigiosa cattedra; era il sesto di una serie di nomi importantissimi: Heinrich Wölfflin, Wilhelm Pinder, Hans Jantzen, Hans Sedlmayer e Stephan Braunfels. Con la prolusione che intitolò quindi provocatoriamente Das Ende der Kunstgeschichte?, Belting si presentò agli eredi della scuola di Sedlmayer. Dalla produzione artistica si era spostato alla ricerca sulla disciplina stessa che la studia: la storia dell'arte.

Nel saggio del 1998 Belting analizza invece la situazione venutasi a creare per la storia dell'arte in Germania al di là della fine della Seconda Guerra Mondiale. La questione della «germanicità» della storia sia di alcuni artisti (Albrecht Dürer o Hans Holbein, ad esempio) era divenuta centrale all'interno di un recupero delle radici storiche e culturali di questo paese. Nel 1995 Belting stesso torna sul suo testo del 1983. In The End of the Art History: A Revision after Ten Years afferma che nessuna lettura della storia è mai assoluta e che la revisione non è un episodio occasionale ma un metodo per rileggere e riaprire questioni e metodologie che non è possible mai stabilire una volta per tutte. Tuttavia la storia dell'arte è una disciplina che richiede norme e parametri, affinché lo studio porti a risultati e dimostri con i fatti percorsi e andamenti dell'arte.

Percorrendo invece alcuni degli studi che hanno posto la storia dell'arte al centro delle ricerche, troviamo una serie di testi che costituisce un excursus fondamentale del dibattito sulla disciplina negli ultimo venti anni. Nel 1994 Art
History's History di Vernon Hyde Minor e, nel 1995, il libro di Eric Fermie, Art History and Its Methods (1995) conducono il dibattito ancora verso altre prospettive.

Nella premessa di The Subjects of Art History. Historical Objects in Contemporary Perspective, pubblicato nel 1998 da Cambridge University Press, si afferma invece che «la storia dell'arte come molti altri campi umanistici, è entrata in una fase post-epistemologica», che oramai «'idea di una estetica di valore universale e la validità della ricerca storica è oggetto di domande», che inoltre la storia dell'arte non è «un campo di studi unitario» e che nuove interrogazioni sono necessarie (Cheetham, Holly y Moxey, 1998).

Nel 1999 esce il libro di Griselda Pollock, Differencing the Canon. Feminist desire and the Writing of Art Histories (Pollock, 1999). Da una prospettiva «femminista» la Pollock si interroga sul "canone» ovvero se il tradizionale canone dei «vecchi maestri» possa essere rifiutato, sostituito o trasformato. II canone non può essere distrutto, dice tuttavia la Pollock: deve essere differenziato.

Il libro di Mieke Bal Quoting Caravaggio: Contemporary Art, Preposterous History, sempre del 1999, può essere considerato una ipotesi interessante di una diversa modalità di citare e associare tra loro opere del presente e del passato. Mieke Bal cerca la sopravvivenza nella storia dell'arte di figure (parole e immagini) tra opere appartenenti a epoche lontane (Bal, 1999). Una figura, una iconografia presente in un'opera contemporanea, può rimandare non a testi (per essere compresa) ma a altre immagini e figure. Da questo avvicinamento di motivi ricorrenti, che esulano dalle convenzionali contestualizzazioni storiche legate ai contesti, agli stili presenti in un'epoca o che caratterizzano l'opera di un artista, nascono nuovi significati, prodotti intertestualmente come nel considerare aspetti dell'opera di Caravaggio che precorrono aspetti dell'opera di un'artista come Ana Mendieta. L'opera d'arte contemporanea è un continuo lavoro (reworking) sul passato, osserva Mieke Bal. È il passato, non il presente, che continuamente si rinnova e si ricostruisce attraverso il presente, che lo osserva e lo rilegge da altri punti di vista. II significato di un'opera non è quindi mai presente totalmente nell'opera: è un «evento», ci dice ancora Mieke Bal, che si produce mettendo in transito le adiacenze, le citazioni, i silenzi che acquisiscono nuova voce proprio negli apparenti impossibili ma invece importanti incontri tra immaginazione, punto di vista che guarda (con i suoi affetti e desideri) e storia. 
Devant le temps. Histoire de l'art et anachronisme des images di Georges Didi-Huberman esce nel 2000. Didi-Huberman considera, a partire dal particolare di Beato Angelico nel registro inferiore del corridoio orientale del Convento di San Marco a Firenze, come questo e tante altre immagini ben visibili «non fosse stato sino ad allora osservato, né interpretato e neppure intravisto nella sterminata letteratura scientifica dedicata alla pittura del Rinascimento». Per Didi-Huberman la questione è epistemologica e culmina nella domanda «che cosa, nella storia dell'arte come disciplina, come ordine del discorso ha mantenuto una tale situazione di accecamento» (Didi-Huberman, 2000).

Nel 2002 il Getty Research Institute diede avvio a un progetto di traduzione, con l'intenzione di produrre traduzioni in inglese dei più importanti testi storici di ogni parte del mondo.

Nel 2003 il Journal of Visual Culture pubblicava gli interventi di nove studiosi che rispondevano a quanto Mieke Bal aveva sostenuto a proposito di una attuale fine dei confini delle discipline. II dibattito su una fine o su una revisione dei tradizionali confini disciplinari (arte, antropologia, linguistica, studi sull'immagine, semiotica) continuò in riviste internazionali tra le quali Representations, Res, Critical Inquiry, Kritische Berichte e, in Italia, L'uomo nero. Queste ricerche sono poi proseguite su altre riviste assumendo pian piano una identità specifica: gli studi visuali, i cultural studies, l'arte nella prospettiva degli studi postcoloniali. Questi sono diventati, soprattutto negli Stati Uniti, in Inghilterra e nel Nord Europa (meno in Francia), non soltanto settori ma punti di vista critici a partire dai quali si sono formulate letture e riletture di interi periodi della storia dell'arte. Le riviste Journal of Visual Culture, Parallax, Invisible Culture, Third Text, Screen hanno dedicato ampio spazio a queste prospettive.

All'University College Cork, tra il 2004 e il 2006, fu messo poi insieme un database di circa ottocento istituzioni internazionali accademiche che presentavano dipartimenti di storia dell'arte, centri o programmi di storia dell'arte. Storici dell'arte della Finlandia, della Giordania, da Singapore, dalla Germania e Danimarca, avevano trasmesso gli elenchi delle istituzioni nei loro paesi che offrivano corsi di storia dell'arte. Emergevano dati interessanti e numeri di dipartimenti sparsi nel mondo: in Irlanda e Regno Unito 97; in Europa 193; in Germania 50. L'Europa dell'Est presentava pochi dipartimenti così come anche l'Europa meridionale: in Cecoslo- vacchia 2; in Romania 2; in Bulgaria 2. Gli interventi di una tavola rotonda organizzata nella primavera del 2005, sempre presso I'University College Cork., a cura di John Elkins, furono pubblicati in Is Art History Global? (Elkins, 2005).

Nel 2007 usciva il libro Making Art History. A Changing Discipline and its Institutions, a cura di Elizabeth C. Mansfield, per le edizioni Routledge: la prima parte era dedicata a come il "canone» nella storia dell'arte poteva essere ripensato in rapporto agli attuali processi globali, non per negare la sua importanza decisiva, ma per analizzare i processi culturali e sociali che contribuiscono alla sua costruzione e, con essa, alla legittimazione e storicizzazione di artisti, movimenti, tendenze nella storia dell'arte; la seconda parte prendeva in considerazione «i soggetti della storia dell'arte»; la terza, le relazioni tra storia dell'arte e università affrontando le questioni degli obiettivi della formazione accademica, dell'identità della professione dello storico dell'arte ma anche delle attuali trasformazioni e dei limiti dell'università oggi; la quarta parte poneva invece l'accento sulle connessioni tra storia dell'arte e istituzioni, ovvero su come queste rispondano a politiche culturali e come contribuiscano alla loro costruzione e diffusione (Mansfield, 2007).

Nel 2010 un altro testo torna su tali questioni. After the Event. New Perspectives on Art History, a cura di Charles Merewether e John Potts, è un libro che cerca di offrire punti di vista «per espandere o sfidare alcuni dei paradigmi prevalenti della storia dell'arte euro americana». Lo studio delle radici alla base della storia dell'arte nel mondo occidentale sembra essere diventato forse il nodo fondamentale di un dibattito molto acceso e oramai argomento di una letteratura già molto vasta, soprattutto da parte dei paesi stessi che tale modello avevano costruito. Si tratta infatti di una letteratura di ambito inglese e statunitense. Nel libro del 2010 molta attenzione è indirizzata allo studio di contesti che erano rimasti al di fuori dalle storia dell'arte più nota. La questione della modernità diventa il punto critico non soltanto per analizzare il sistema della periodizzazione ma anche per confrontare storie diverse e sistemi di periodizzazione non comparabili, esito di vicende diverse della storia e della cultura all'interno di una determinata nazione.

Le ipotesi di storicizzazione hanno proceduto secondo criteri epistemologici diversi, per provare a pensare altre costruzioni della storia e anche per risalire a quanto la storia sembra dover escludere: la memoria, i paradossi del tempo, i dati che possono mettere in crisi sistemi di lettura o storiciz- 
zazione che cercano l'unitarietà, le convergenze, lo «stile» di un artista o di un'epoca, trascurando o marginalizzando ciò che entro queste premesse non può essere compreso. Se questi fatti sommersi o rimasti silenziosi tornano tuttavia a attivare la propria presenza, semmai attraverso condizioni che li richiamano, che li fanno rivivere nel presente, cosa accade?

In un suo testo dal titolo Trouble in the archive, ancora la studiosa Griselda Pollock affermava:

L'uso del termine «archivio» insiste sul fatto che quello che noi studiamo come storia non è una accumulazione del passato, conservato nelle biblioteche per noi al fine dello studio. Ciò che è preservato, conservato e classificato come materiali per lo studio della storia e del patrimonio culturale è stato collocato lì in accordo agli interessi sociali e ai desideri di specifiche classi sociali, culture e generi. La biblioteca e il museo non sono luoghi innocenti della conservazione; sono sempre testi costruiti in accordo agli interessi e ai bisogni di certi gruppi. Questo archivio canonizzato forma poi attivamente il presente per noi (Pollock, 2001: 31).

Più avanti precisava:

Il termine archivio ha anche un'altra implicazione. Ci rende coscienti come storici, richiedendoci un criterio necessario per la selezione in quanto noi ci imbattiamo in materiali che hanno diversi passati: un momento storico di produzione, un momento storico di consumo, un momento storico che entra nel discorso storico, nel museo, nel canone, nell'aula, nei nostri patrimoni culturali; in una miriade di strutture discorsive e nella nostra formazione di «soggetti» che fanno o studiano l'arte, o anche in certi culture formate proprio da cosa e da cosa non è presente nell'archivio (Pollock, 2001: 32).

La storia dell'arte è dunque sempre una forma di rappresentazione della storia: come un archivio non racconta che una narrazione, tralasciando le molte altre storie possibili.

Nel 1999, Gayatri Chakravorty Spivak, nel suo Critica della ragione postcoloniale. Verso una storia del presente in dissolvenza, avanzava il punto di vista che è impossibile trovare nei «documenti» e negli «archivi» (nei quali lo «storico» crede di poter trovare) tutte le informazioni per costruire la storia: è impossibile perché nei documenti e negli archivi non possiamo trovare quel «soggetto silenzioso» che in diverse epoche, subordinato a apparati ideologici o di potere, ha visto distorcere, annullare o marginalizzare la sua «voce», fino a renderla quasi muta. Di questo «soggetto silenzioso» cosa e come possiamo trovare negli archivi? Come ricostruirne la presenza? Dove cercarla? Inoltre, sono i nessi e le relazioni di casualità (cause e effetti, giochi di influenze, percorsi tra precursori e successori), presentati come gli unici possibili, che cancellano ogni dubbio e incertezza sulla possibilità che quei fatti siano veramente quanto avvenuto. La dimenticanza o l'oblio di tutto ciò che è stato ritenuto marginale, resta sommerso (Spivak, 1999).

Nel 1997 era uscito il libro Caught By History: Holocaust Effects in Art, Literature, and Theory di Ernst van Alphen. Attraverso una testimonianza quasi autobiografica di come proprio in un individuo nato nel 1958 il racconto della seconda Guerra mondiale e della tragicità dell'Olocausto siano per molto tempo risultati racconti fatti per ricevere una sola e sempre identica risposta, Ernst Van Alphen passa con questo libro a una riconsiderazione di quell'epoca, arrivando a parlare di Holocaust effect nell'arte e nella cultura più recenti, concetto profondamente diverso da quello di Holocaust representation ovvero dalla «rappresentazione» dell'Olocausto (Alphen, 1997: 106).

L' «effetto» è un concetto dinamico che nasce non dal confronto con le rappresentazioni dell'Olocausto ma con l'esperienza che se ne può fare, attraversando gli effetti della storia dove essi si ripresentano, dove rivivono nel presente. L'esperienza di questi effetti è una esperienza performativa dove memoria, immaginazione e storia si incontrano, nelle adiacenze o, meglio, nelle tensioni attraverso le quali il passato torna a ripresentarsi nel presente.

Il lavoro dello storico, tuttavia, difficilmente o raramente utilizza la sua «scoperta» come un dato che potrebbe mettere in crisi quanto studiato, storicizzato e legittimato fino alla sua ricerca. II criterio della «eucronia», come ha ben dimostrato Georges Didi-Huberman resta una premessa per lo storico che non farà che ricondurre in temporalità credute punti fermi della storia, i dati, i documenti, gli ultimi «ritrovamenti» studiati. È proprio per cercare di interrogare questo metodo che Didi-Huberman, fa dunque l'ipotesi dell' "anacronismo», e arriva a porre la domanda: «la storia dell'arte è di per sé una disciplina anacronistica?» (Didi-Huberman, 2007: 24).

La domanda che sembrerebbe nascere è allora questa: se Mieke Bal parla di una storia «preposterous», in cui la vita- 
lità dell'immagine, «sopravvivendo» alla sua epoca e al contesto che l'ha prodotta, intrattiene relazioni e effetti con altre immagini di altri tempi e storie, divenendo ogni volta il suo significato ripensato e trasformato a seconda di come tale vitalità continua a produrre i suoi effetti; se Didi-Huberman parla di una storia dell'arte in cui le adiacenze, l'azione delle distanze temporali (un'opera o un'immagine appartenenti a contesti, a epoche diverse, anche lontane nel tempo) e dei «ritmi alterni» tra quelli che chiama «i momenti di prossimità - empatici, intempestivi, inverificabili - e i momenti di distanziamento critico", ovvero quelli attraverso i quali lo storico verifica e afferma le sue conclusioni"; se come già Walter Benjamin affermava «la specificità storica» di un'opera d'arte non è da rintracciare all'interno del racconto fatto di cause e effetti che pretenderebbe di comprenderne la sua posizione in una narrazione già fatta, ma nelle «connessioni tra diverse opere d'arte che sono atemporali e tuttavia non mancano di rilevanza storica» (Benjamin,1978: 72), allora, è possibile pensare una storia che restituisca la vitalità alle opere, ai documenti, ai dati già verificati ma anche ad altri dati ancora da inserire in possibili altre narrazioni, sottraendo prima tutti questi dati che crediamo acquisiti dalla «storia» a cui sembrano inevitabilmente appartenere, per far saltare le simmetrie e le «eucronie», per restituire quei dati alla loro iniziale efficacia storica ma anche di eventi, in grado di suggerire altre letture, altri racconti e non interpretazioni finalizzate a costruire nessi o ipotesi che anziché far vivere quel dato, non fanno che devitalizzarlo in griglie, schemi, strutture discorsive già fatte, che in quanto tali non possono comprendere quel che proprio i fondamenti teorici e critici di partenza non possono accogliere.

Se ad esempio si tentasse dunque una riscrittura della storia dell'arte della seconda metà del XX secolo quali problemi nascerebbero? Come utilizzare le storie già esistenti? Come invece inserire le storie che sono restate silenziose e che ancora lo sono, ad esempio la storia dei paesi dell'Europa dell'Est, o le storie di molte sperimentazioni che nel meccanismo selettivo di una storia costruita su parametri di successo, mercato, collezionismo sono restate ai margini o addirittura taciute? Partendo da un punto di vista radicato nel presente, all'inizio del XXI secolo, la scrittura di una storia possibile della seconda metà del XX secolo si presenta assai ampia: una storia che coinvolge l'intero pianeta, e della quale si potrebbe tentare una storia più ampia, a partire dalla metà del secolo, ovvero quando di molti Paesi del mondo si ignoravano (nelle storie più conosciute e che sono alla base della preparazione accademica) sia le specificità che le audaci e forse talvolta ancora più radicali sperimentazioni, proprio perché al di fuori di molte strategie della storia invece già sistematizzata e avviata. Se teniamo fermo il dato che una storia generale, una storia che sia davvero la costruzione di tutti i dati o che possa essere la convergenza di tutto quanto è stato trovato, non può più essere fatta; se una storia è sempre una rappresentazione tra le molte altre possibili, l'idea che la storia costruisca nel tempo i propri risultati e che tali dati siano continuamente confermati e riconfermati da quanto, in seguito, sarà trovato e usato come ulteriore conferma e validità della veridicità del passato, non può essere più ritenuta la sola strada possibile. Prima di tutto è in crisi l'idea di una sola storia dell'arte. Piuttosto che pensare di colmare le lacune e di poter inserire, aggiungere tutti i dati mancanti, è lo stesso ordine discorsivo che ha selezionato i fatti, costruendo tra essi alcuni percorsi, che non può accettare ciò che è stato marginalizzato, taciuto o escluso. Non si tratta neanche di fare una storia del «sommerso» della storia: anche questa ipotesi, posta in questi termini, non sarebbe che un percorso parallelo, forse costruito secondo i medesimi criteri di cause effetti, di precursori e successori alla base di molte storie che conosciamo.

Si può tentare di procedere attraverso le discontinuità, sia storiche che geografiche, affiancando ciò che è tornato in momenti diversi di questo periodo (dal 1945 a oggi), procedendo attraverso le adiacenze tra le differenze e non tra le identità orami chiuse nei movimenti artistici, nelle sterili e talvolta molto approssimative successioni di fatti che in realtà sono nati da situazioni, contesti culturali, sociali assai diversi. Queste adiacenze o discontinuità indicano più percorsi paralleli, dimostrano come una storia onnicomprensiva, oggi, sia forse impossibile. In questo andare e tornare, da oggi all'indietro, da un punto della storia verso altri molteplici punti che nello stesso tempo si costruivano non più su una linea di sviluppo, ma nell'incrocio di una fitta rete di relazioni visibili o invisibili, ci sono non singoli aspetti della storia ma macrostrutture di essa che rivelano oggi la fragilità stessa di percorsi creduti per molto tempo assoluti.

Prima tra queste macrostrutture: la modernità. II nuovo ordine mondiale che Samuel P. Huntington descrive nel suo The Clash of Civilizations and the Remaking of World Order (1996) ipotizza che: 


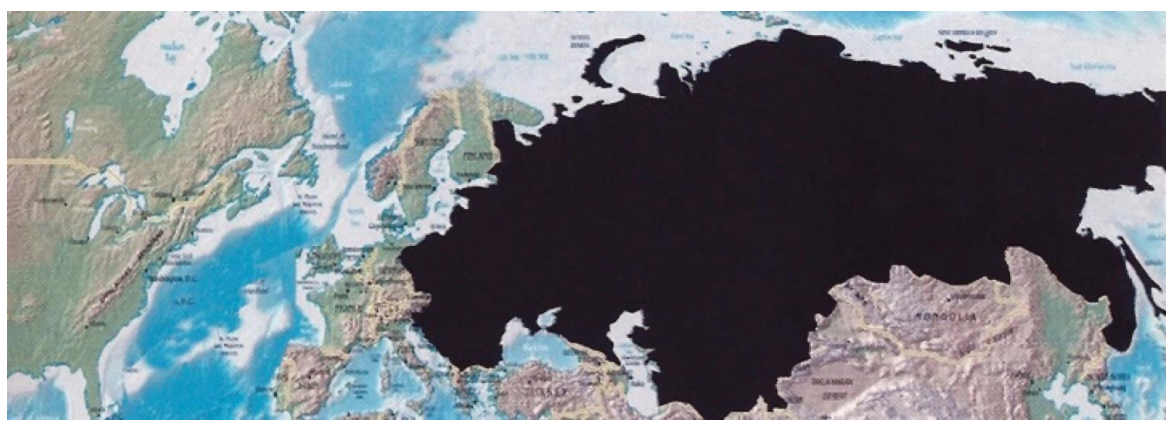

1. Irwin, East Art Map, 2006

Modernizzazione, in definitiva, non significa necessariamente occidentalizzazione. Le società non occidentali possono modernizzarsi, e l'hanno fatto, senza abbandonare la propria cultura e senza adottare in blocco valori, istituzioni e costumi occidentali (Huntington, 2015: 104-105).

Anthony Giddens in The Consequences of Modernity ha affermato che la modernità non ha mostrato solo lati positivi e aperto opportunità ma ha mostrato soprattutto un lato oscuro, una serie di rischi che prima di questa fase della storia era stato difficile cogliere: il sistema capitalistico, il potere politico, il potere militare sono conseguenze della modernità che oggi devono essere riconsiderati da questo punto di vista (Giddens, 1990).

Per altro verso, Timothy J. Clark alla fine degli anni Novanta, in Farwell to an Idea: Episodes from a History of Modernism, considera la modernità alla sua fine seppur con la malinconia di una perdita definitiva (Clark, 1999).

Svetlana Boym nel 2001 ha parlato di «off-modern»: diversi modernismi e condizioni non lineari del loro sviluppo hanno aperto non ad una ma a molteplici direzioni per seguire quello che è stata e ciò che ha significato la modernità nel mondo. Non una ma più modernità si sono sovrapposte, incrociate, hanno vissuto parallelamente, in una rete di relazioni che non possono essere oggi sottovalutate (Boym, 2001).

Una recente mostra Les promesses du passé inauguratesi al Centre Pompidou nel 2010 proponeva la rilettura della storia dell'arte nei paesi dell'Europa dell'Est. II progetto nato pressoché negli stessi anni di un altro ampio progetto di revisione della storia dell'Europa a partire dalla situazione che durante la Guerra fredda aveva oscurato l'attenzione su un'intera metà del continente, si tratta del progetto degli artisti del gruppo Irwin East Art Map [1]. La mostra articolava nelle sale del Centre Pompidou una storia che difficilmente fino ad allora era stata poco conosciuta.

«La cultura moderna era caratterizzata dalla tensione tra nazionale e internazionale, la cultura postmoderna è globalizzata, funziona nel quadro delle dottrine multiculturali e opta per un altro vocabolario» affermava Piotr Piotrowski in un testo del catalogo della mostra (Piotrowski, 2006: 215).

In questa prospettiva è l'identità, sempre secondo Piotrowski che viene separata ad esempio dal concetto di nazione per tornare a configurarsi nella molteplicità delle differenze anche sessuali, locali, etniche. La marginalizzazione dell'identità in nome dell'utopia universale dell'unità era stato anch'esso un risultato della modernità occidentale. In questo senso molti altri concetti si modificano: la transnazionalità è altra cosa dall'internazionalità, la contemporaneità dalla modernità, l'universalità dall'intreccio complesso delle differenze.

East Art Map, così il nome del progetto di Irwin, a partire dal 2001, ha realizzato un'ampia piattaforma nel web continuamente aggiornata, per «ricostruire la storia dell'arte nell'Europa orientale tra il 1945 e oggi sfidando i sistemi chiusi della interpretazione e della valutazione». Se infatti «la mappa dell'arte occidentale è largamente presente nelle conoscenze di quasi tutti seppur nelle linee fondamentali di base [...] non così è per quello che riguarda l'Europa dell'Est dove non esiste una struttura trasparente di una sua storia dell'arte generale» (Irwin, 2006).

Cambiare il punto di vista, non partendo da un discorso convenzionale della divisione Est-Ovest, ma focalizzando 
l'attenzione su una modalità del tempo e un ritmo ineguale della storia diventava l'indicazione critica della mostra che piuttosto che considerare le sole opere dell'Europa dell'Est prediligeva lo sguardo sulla coesistenza di diverse narrazioni, in differenti parti del mondo. Queste narrazioni rivelavano storie e conflitti, dimenticanze e gerarchie, lacune e configurazioni di potere negli stessi meccanismi della storia dell'arte.

Se per altro verso riconsideriamo così le storie dell'arte che datano prima del 1989 e quelle che invece nascono dopo questa data, notiamo quindi una profonda differenza. Tale differenza porta a far emergere prima di tutto una serie di problemi che il dibattito internazionale, anche italiano, cerca di affrontare per trovare alcune risposte e per ripensare i metodi di una disciplina viva e importante come la storia dell'arte. La differenza è il risultato di una diversa impostazione, non soltanto metodologica, ma emersa nella più ampia prospettiva storica, geografica, politica che si configura dopo il 1989. Quindi quelli che si pensavano i margini del mondo all'interno dei quali la storia dell'arte era proceduta, sono saltati e altri orizzonti si sono affacciati. Non è soltanto un mutamento di paesaggio. Mettendo in relazione epoche e storie, paesi e tradizioni, culture anche molto lontane, così come sta avvenendo a livello globale, questi e altri parametri, creduti imprescindibili, si sono rivelati insufficienti e con essi gli strumenti critici e ermeneutici attraverso i quali la storia, anche dell'arte, è costruita.

\section{Bibliografia}

ALPHEN, Ernst van (1997), Caught By History: Holocaust Effects in Art, Literature, and Theory, Stanford University Press, Stanford.

BAL, Mieke (1999), Quoting Caravaggio: Contemporary Art, Preposterous History, Chicago University Press, Chicago.

BELTING, Hans (1983), Das Ende der Kunstgeschicute. Eine Revision nach zehn Jahren, C.H. Beck, Múnich.

— (1998), The Germans and their Art, Yale University Press, Yale.

BENJAMIN, Walter (1978), Lettere 1913-1940, Einaudi, Torino.

BOYM, Svetlana (2001), The Future of Nostalgia, Basics Books, New York.

CHEETHAM, Mark A., HOLLY, Michael Ann y MOXEY, Keith (1998), The Subjects of Art History. Historical Objects in Contemporary Perspective, Cambridge University Press, Cambridge.

CLARK, Timothy J. (1999), Farwell to an Idea: Episodes from a History of Modernism, Yale University Press, New Haven-London.

DIDI-HUBERMAN, Georges (2000), Devant le temps. Histoire de l'art et anachronisme des images, Éditions de Minuit, Paris; tr. it. (2007), Storia dell'arte e anacronismo delle immagini, Bollati Boringhieri, Torino.

ELKINS, John (2005), Is Art History Global?, Routledge, New York-London.

FERMIE, Eric (1995), Art History and Its Methods, Phaidon Press, London.

GIDDENS, Anthony (1990), The Consequences of Modenity, Polity Press, Cambridge; tr. it. (1994), Le conseguenze della modenità, II Mulino, Bologna.

HYDE MINOR,Vernon (1994), Art History's History, Prentice Hall, Boston.

HUNTINGTON, Samuel P. (1996), The Clash of Civilizations and the Remaking of World Order Penguin Books, London; tr. it. Lo scontro delle civiltà e il nuovo ordine mondiale (2015), Garzanti, Milano.

IRWIN (2006), East Art Map: Contemporary Art and Eastern Europe, Afterall Books, London.

MACEL, Christine y PETRESIN-BACHELEZ, Nataša (2010), Les Promesses du passé, Centre Pompidou, Paris.

MANSFIELD, Elizabeth C. (2007), Making art history. A changing discipline and its institutions, Routledge, London-New York.

MEREWETHER, Charles y POTTS, John (2010), After the Event. New Perspectives in Art History, Manchester University Press, Manchester. PIOTROWSKI, Piotr (2006), East Art Map: COntemporary Art and Eastern Europe, Irwin, Londres.

POLLOCK, Griselda (1999), Differencing the Canon. Feminist Desire and the Writing of Art Histories, Routledge, London-New York.

- (2001), Trouble in the Archive, in Looking Back to the Future. Essays on Art, Life and Death, G+B Arts, London.

SPIVAK, Gayatri Chakravorty (1999), A Critique of Postcolonial Reason. Toward a History of the Vanishing Present, Harvard University Press, Cambridge-Massachusetts, London; tr. it. (1999), Critica della ragione postcoloniale. Verso una storia del presente in dissolvenza, Meltemi, Roma. 